\title{
Hard X-ray and Optical Activity of Intermediate Polars
}

\author{
Rudolf Gális ${ }^{1}$, Ladislav Hric ${ }^{2}$ and Emil Kundra ${ }^{2} \dagger$ \\ ${ }^{1}$ Department of Theoretical Physics and Astrophysics, Institute of Physics, \\ Faculty of Sciences, P. J. Šafárik University, Park Angelinum 9, 04001 Košice, Slovakia \\ email: rudolf.galis@upjs.sk \\ ${ }^{2}$ Astronomical Institute of the Slovak Academy of Sciences, 05960 Tatranská Lomnica, \\ Slovakia
}

\begin{abstract}
Intermediate polars represent a major fraction of all cataclysmic variables detected by INTEGRAL in hard X-rays. Nevertheless, only $25 \%$ of all known intermediate polars have been was detected in hard X-rays. This fact can be related to the activity state of these close interacting binaries. Multi-frequency (optical to X-ray) investigation of intermediate polars is essential to understand the physical mechanisms responsible for the observed activity of these objects.
\end{abstract}

Keywords. stars: novae, cataclysmic variables, accretion, accretion disks

\section{Introduction}

Cataclysmic variables (CVs) manifest strong activity in the whole spectrum from radio up to $\gamma$-rays. CVs are close binary systems consisting of a hot white dwarf (WD) and red main-sequence star, which fills the volume of its inner Roche lobe and transfers matter to the vicinity of the WD (Warner 1995). The mass transfer between components is a main reason for the observed $\mathrm{CV}$ activity.

According to the strength of the WD magnetic field, the transferred matter is creating an accretion disk or follows magnetic lines and falls to the surface of the WD. In intermediate polars (IPs), the WD magnetic field $\left(10^{4}-10^{6} \mathrm{G}\right)$ is not strong enough to disrupt the disc entirely (as in the case of polars) and simply truncates the inner part of the disc. An accretion flow is channelled down towards the magnetic poles and onto the WD surface.

When the transferred material impacts the WD atmosphere, a shock will form and hard X-ray emission will result from thermal bremsstrahlung cooling by free electrons in the hot post-shock region (King \& Lasota 1979). The broad-band spectra (3-100 keV) of the studied IPs can be well fitted by a thermal bremsstrahlung model with the post-shock temperature of $k T \approx(20-25) \mathrm{keV}$ (Gális et al. 2009).

No significant modulation has been found so far in the 20 - $30 \mathrm{keV}$ light curves (Barlow et al. 2006). Nevertheless, the sample detected by INTEGRAL represented only $25 \%$ of all known IPs (Gális et al. 2009). Some IPs were not detectable even though we had significant exposure time (more than $4 \mathrm{Msec}$ ) for these sources. This fact can be related to the activity state of these interacting binaries.

We analysed all available observational data from INTEGRAL/IBIS for IP V1223 Sgr (Gális et al. 2009). Our analysis showed that the fluxes of this object are long-term variable, mainly in (15 - 25) keV and (25-40) keV bands. Moreover, this hard X-ray variability is correlated with changes in the optical spectral band. Our analysis revealed a deep flux drop around MJD $\approx 53650$ observed in both X-ray and optical bands for this IP.

$\dagger$ This study was supported by the project of the Slovak Academy of Sciences VEGA Grant No. $2 / 0078 / 10$. 

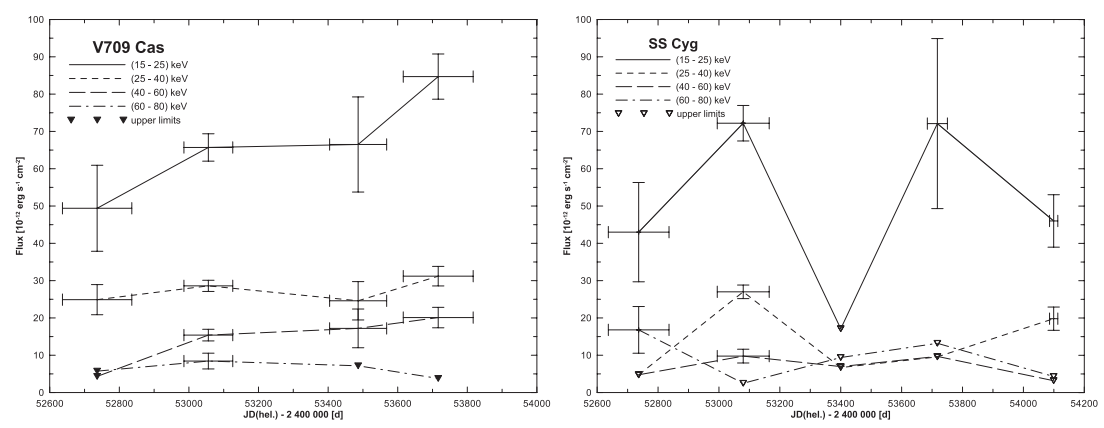

Figure 1. INTEGRAL/IBIS flux curves of V709 Cas (left panel) nad SS Cyg (right panel) in the corresponding energy bands. The arrows represent $3 \sigma$ upper limits.

\section{Observations, analysis and results}

We used all publicly available observational data from INTEGRAL/IBIS to study possible variability of V709 Cas and SS Cyg in hard X-rays. Observational data used in our analysis were processed by INTEGRAL's Offline Standard Analysis Package OSA7.

V709 Cas was recognized as an IP following its detection in the ROSAT All Sky Survey. The broad-band $(3-100) \mathrm{keV}$ spectrum from INTEGRAL was well fitted by a thermal bremsstrahlung model with the temperature of $k T=24.4_{-1.4}^{+1.5} \mathrm{keV}$ (Gális et al. 2009).

SS Cyg is an optically bright IP observed to undergo outbursts every $\approx 40$ days, characterized by an increase in optical magnitude from $\mathrm{V} \sim 12$ to $\mathrm{V} \sim 8$. Comparison of the INTEGRAL/IBIS flux curve with the optical light-curve (validated AAVSO data) over part of the IBIS survey period suggested a strengthening of the $20-30 \mathrm{keV}$ flux during optical quiescence (Barlow et al. 2006).

Our analysis of all available observational data of V709 Cas and SS Cyg showed that these sources are detectable up to $100 \mathrm{keV}$. The hard X-ray fluxes are not persistent. The flux curves indicate that the brightness of V709 Cas increased by a factor $\approx 2$ from MJD 52700 to MJD 53700 in the (15-25) keV energy band (Fig. 1, left panel) and the brightness of SS Cyg increased by a factor $\approx 2$ in the $(15-25) \mathrm{keV}$ and $(2540) \mathrm{keV}$ energy bands during the optical quiescence phases (Fig. 1, right panel).

\section{Conclusions}

The significant part of the optical emission from IPs is produced by a hot spot, where the matter from the donor star interacts with the outer rim of the accretion disk. X-ray emission is produced by the interaction of the accreting matter with the WD surface. So, the emission in both optical and X-ray bands is related to the mass transfer and therefore observed variations are probably caused by changes in the mass accretion rate.

We are preparing the photometric campaign to obtain long-term homogenous observations (to cover whole activity cycles) as well as sets of observations with high time resolution (to detail coverage of orbital cycles) of selected CVs, mainly as a follow up of the INTEGRAL observations. Simultaneous analysis of multi-frequency observation (from optical to X-ray) allows complex study of the physical mechanism related to the mass transfer in these interacting binaries.

\section{References}

Barlow, E. J., Knigge, C., Bird, A. J. et al., 2006, MNRAS, 372, 224

Gális, R., Eckert, D., Paltani, S., Münz, F., \& Kocka M. 2009, Baltic Astronomy, 18, 321

King, A. R. \& Lasota, J. P. 1979, MNRAS, 188, 653

Warner, B. 1995, Cataclysmic variable stars, Cambridge University Press, Cambridge 